\title{
Антикорреляция интенсивности стимулированного пикосекундного излучения GaAs и характерного времени остывания носителей заряда
}

\author{
(C) Н.Н. Агеева, И.Л. Броневой , Д.Н. Забегаев, А.Н. Кривоносов \\ Институт радиотехники и электроники им. В.А. Котельникова Российской академии наук, \\ 125009 Москва, Россия \\ I E-mail: bil@cplire.ru
}

Поступила в Редакцию 10 июля 2019 г.

В окончательной редакции 5 августа 2019 г.

Принята к публикации 20 августа 2019 г.

Во время мощной пикосекундной оптической накачки в тонком слое GaAs возникает интенсивное стимулированное пикосекундное излучение. Обнаружено, что, во-первых, максимальная интенсивность излучения уменьшается при увеличении диаметра луча накачки (плотность энергии накачки фиксирована). Во-вторых, эта зависимость антикоррелирует с зависимостью от диаметра характерного времени релаксации излучения. Это время связано с характерным временем остывания носителей заряда, замедляемым из-за разогрева носителей излучением. В итоге выявляется указанная в заглавии автокорреляция.

Ключевые слова: стимулированное пикосекундное излучение, остывание носителей заряда, насыщение усиления, закон Бугера.

DOI: 10.21883/FTP.2020.01.48764.9209

\section{1. Введение}

Собственное стимулированное (усиленное спонтанное) пикосекундное излучение возникает в тонком слое $\mathrm{GaAs}$ во время его оптической пикосекундной накачки. Для краткости будем далее называть его излучение. Накачка носителей заряда одновременно создает просветление слоя, т.е. увеличение его прозрачности. В работе [1] представлена релаксация излучения и просветления при спаде накачки и после ее окончания. Релаксация просветления отображала преимущественно релаксацию плотности носителей заряда. Характерное время релаксации просветления и излучения оказалось связанным с характерным временем релаксации температуры носителей $\tau_{T}$. Это стало предпосылкой для описываемого далее исследования связи интенсивности излучения со временем $\tau_{T}$. Оно опиралось на изменение интенсивности излучения и времени $\tau_{T}$ при увеличении диаметра луча накачки $D$. Обнаруженное изменение интенсивности излучения оказалось при этом еще и противоположно закону Бугера для активной области, выполняемому в линейной оптике. Заметим, что изменение интенсивности излучения при увеличении длины активной области при так называемом насыщении усиления, т.е. когда излучение влияет на его собственное усиление, анализировалось ранее, например, в работах [2,3]. Но это делалось для стационарного излучения и других условий, отличных от наших, и в значительной степени математически. Соответственно в тех работах не было обнаружено эффекта, описываемого в настоящей работе.

\section{2. Эксперимент}

Опыты проводились при комнатной температуре $T_{R}$ на пикосекундном лазерном спектрофотохронометрическом комплексе с автоматической измерительной системой (рис. 1). Комплекс состоит из: задающего YAG-лазера с диодной накачкой PL PDP1-300; системы усилителей лазерного импульса, накачиваемых источниками питания серии 700ТС; удвоителей частоты светового импульса (кристаллов КDP); двух параметрических генераторов света на $\mathrm{LiNbO}_{3}$ с температурной перестройкой длины волны - один для генерации накачивающего, второй - зондирующего или др. импульсов длительностью (FWHM - full width at half maximum) $\approx 10$ пс с линейной поляризацией света; спектрофотохронометрической системы. Наиболее важными компонентами последней являются: двойной спектрограф SpectraPro-2500i, используемый как для спектральных измерений, так и (в режиме вычитания дисперсии) в качестве полосового фильтра длин волн, не искажающего длительность излучения; пикосекундная электроннооптическая камера (ЭОК) PS-1/S1; ПЗС-камеры PIXIS и CoolSNAP:HQ2 для регистрации соответственно спектра излучения и изменения со временем интенсивности спектральной компоненты излучения, пропущенной спектрографом (ПЗС - прибор с зарядовой связью).

Исследуемый образец представлял собой гетероструктуру $\mathrm{Al}_{0.32} \mathrm{Ga}_{0.68} \mathrm{As}-\mathrm{GaAs}-\mathrm{Al}_{0.32} \mathrm{Ga}_{0.68} \mathrm{As}$ с толщиной слоев соответственно 1.2-1.6-1.3 мкм. Слои $\mathrm{Al}_{x} \mathrm{Ga}_{1-x} \mathrm{As}$ являются прозрачными для света, используемого в эксперименте. На поверхностях образца, кроме торцевых, было нанесено антиотражающее покрытие. 


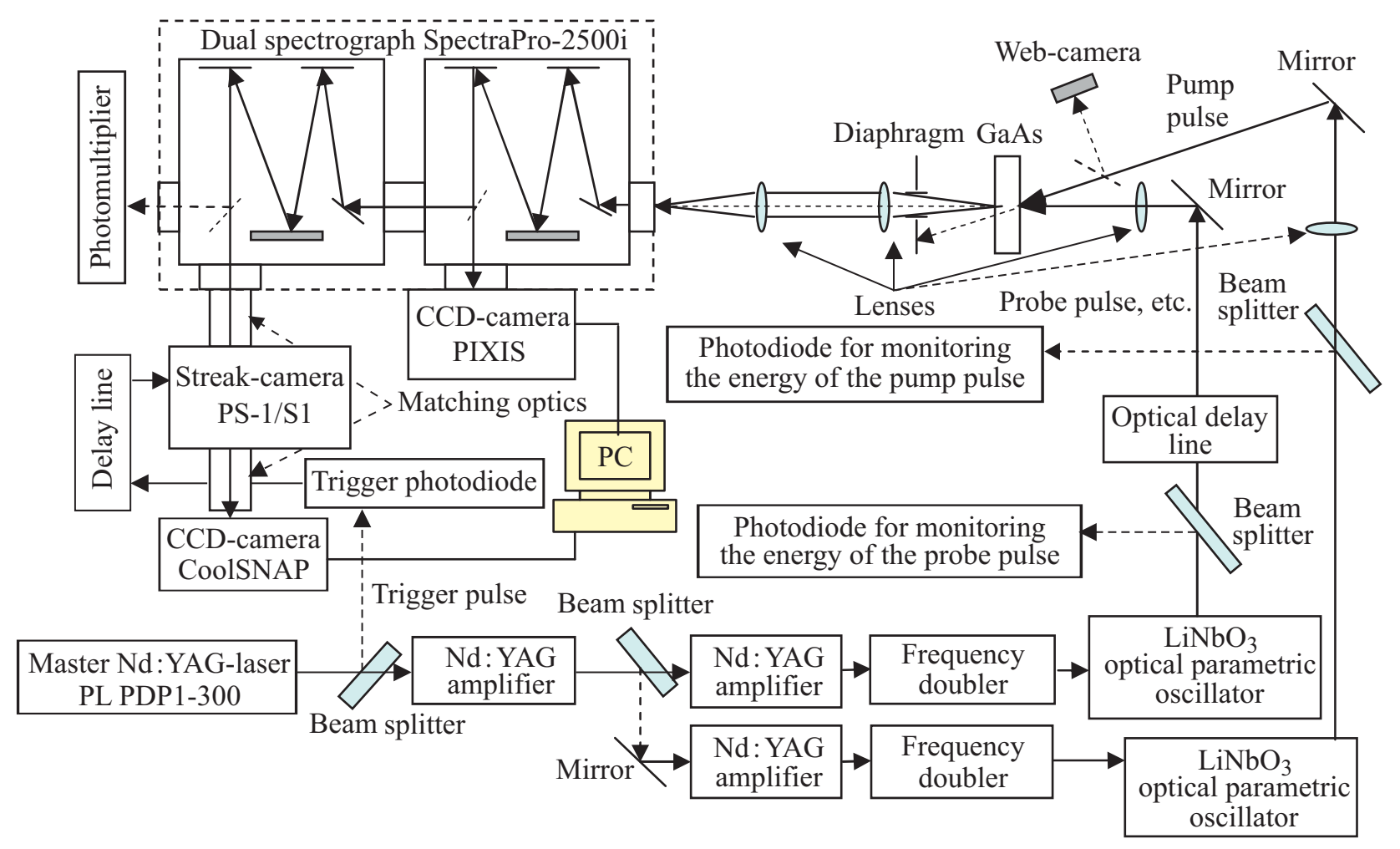

Рис. 1. Схема лазерного пикосекундного спектрофотохронометрического комплекса.

Излучение, возникавшее в оптически накачиваемой области слоя GaAs, усиливалось в ней в направлении вдоль слоя. Диаметр луча накачки $D$ в местоположении образца (FWHM) последовательно изменяли от 0.2 до 1.1 мм. Изменение интенсивности света по сечению луча накачки было приблизительно гауссовым. При всех $D$ плотность энергии накачки $W_{\mathrm{ex}} / V$ поддерживалась неизменной, здесь $W_{\mathrm{ex}}$ - энергия импульса накачки, $V=l \pi D^{2} / 4$ - объем накачанной области, $l-$ толщина слоя GaAs. Благодаря антиотражающему покрытию отражение света накачки от поверхности образца не превышало $2 \%$. Измерение диаметра $D$ проводилось на основе изображения на веб-камере, на которую луч отводился с помощью перемещаемого зеркала (рис. 1). Веб-камера располагалась на том же расстоянии от зеркала, что и исследуемый образец.

Из измерений прозрачности образца для импульсов накачки диаметром $D=0.2$ и 1.1 мм следовало, что плотность поглощаемой в образце энергии импульса накачки отличается на $2 \%$, т. е. практически не зависит от $D$. Скорость стимулированной рекомбинации, по оценкам, намного превышает скорость других типов рекомбинации [1]. С учетом этого при всех $D$ плотность полной энергии излучения $W_{s}$ должна быть одинакова и равна плотности поглощенной энергии накачки за вычетом плотности пороговой энергии накачки. Здесь и далее под плотностью энергии подразумевается средняя энергия по объему $V$.
Такими же, как в работе [1], были измерения: (a) спектра излучения, интегрального по времени, $W(\hbar \omega)$; (б) огибающих импульсов накачки (ex) и излучения $(s)$, интегрального по спектру, и т.д. Результат измерения в реальном времени огибающей импульса накачки и огибающей импульса излучения, представленные в графическом виде, будем называть хронограммами и обозначать $H_{\mathrm{ex}}(t)$ и $H_{s}(t)$ соответственно.

Исследовалась часть излучения, выходившая из накачанной области слоя и распространявшаяся внутри телесного угла $1.2 \cdot 10^{-2}$ стерадиан с осью, ортогональной поверхности гетероструктуры. Поясним, почему указанная часть излучения была выбрана для измерения. Дело в том, что распространение света в веществе должно сопровождаться рассеянием этого света [4]. Достаточным условием для такого явления служит наличие электронов, способных колебаться под действием переменного поля световой волны. В нашем случае это накачанные неравновесные электроны. Если среда оптически однородна, то, в первом приближении, полное взаимное гашение вторичных волн, вызываемых колебаниями электронов, будет происходить для любого направления распространения излучения, не лежащего в плоскости слоя GaAs. Из-за наличия в реальных средах оптических неоднородностей (дефектов) взаимное гашение вторичных волн становится не полным, а частичным, и возникает рассеянный в разных направлениях свет. Пусть $k-$ это доля излучения, которая рассеивалась (выходила из накачанной области слоя 


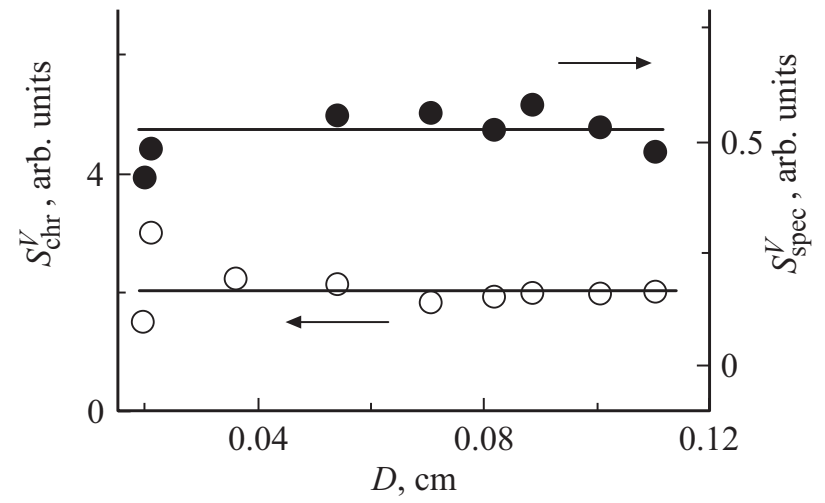

Рис. 2. Площадь под графиком спектра излучения, нормированная на объем $V,-S_{\text {spec }}^{V}$ и хронограммы $-S_{\mathrm{chr}}^{V}$ в функции от диаметра луча накачки $D$.

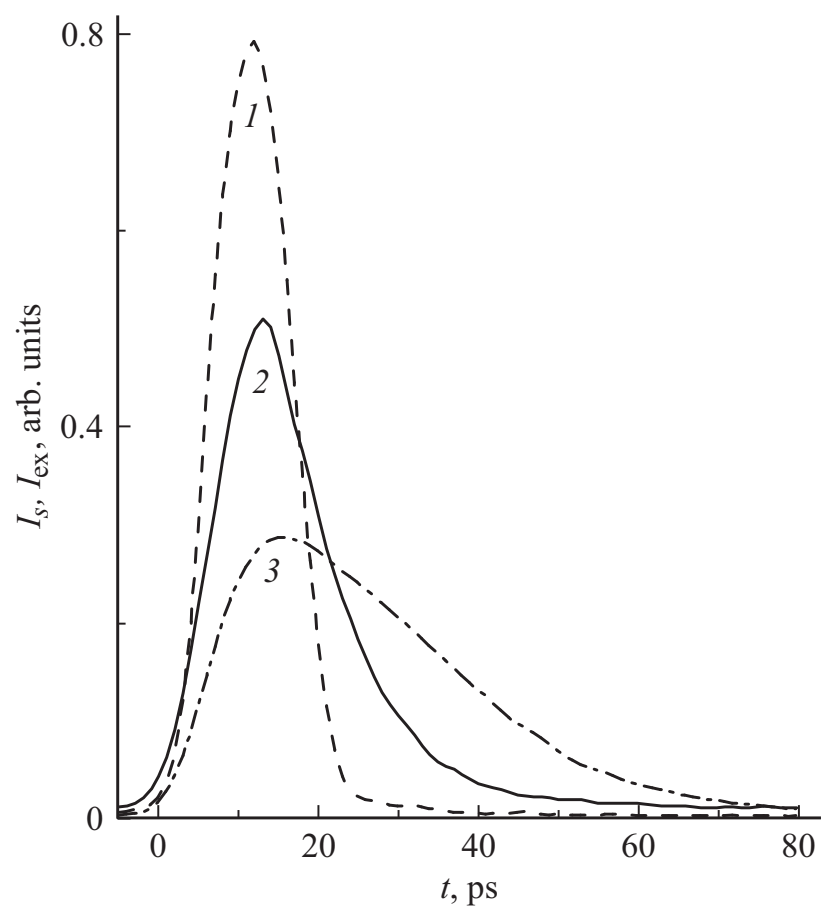

Рис. 3. Нормированные (см. текст) хронограммы накачки $I_{\mathrm{ex}}(t)(1)$ и излучения $I_{s}(t)$ при диаметрах $D=0.21$ мм (2) и $D=1.1$ мм (3).

$\mathrm{GaAs}$ ) ортогонально плоскости слоя и измерялась в эксперименте $(k<1)$. Она была выбрана для измерений потому, что выходит прямо из активной области, не изменяясь из-за поглощения в пассивной области и отражения от торцов образца. То, что излучение, как распространяющееся вдоль эпитаксиального слоя, так и вытекающее через поверхность гетероструктуры из-за ее несовершенства, это одно и то же стимулированное излучение, было доказано в наших предыдущих исследованиях. Доказательства этого были суммированы в работе [1]. Добавим, что диаграмма направленности излучения, измеренная в работе [5], подобна типичной индикатрисе рассеяния, графически показывающей рас- пределение интенсивности рассеянного света по разным направлениям.

Относительно коэффициента $k$ заметим, что он зависит, в частности, от плотности дефектов. Площадь под графиками нормированных на $V$ : (а) спектра излучения $-S_{\mathrm{spec}}^{V}=\frac{1}{V} \int W d(\hbar \omega)$, (б) хронограммы излучения $-S_{\mathrm{chr}}^{V}=\frac{1}{V} \int H_{s} d t$, должна быть пропорциональна $k W_{s}$. Так как в наших условиях $W_{s} \approx$ const, то о пространственной неоднородности плотности дефектов и коэффициента $k$ можно судить по отклонению от константы функций $S_{\mathrm{spec}}^{V}=f(D)$ и $S_{\mathrm{chr}}^{V}=f(D)$ (рис. 2). Площади $S_{\mathrm{chr}}^{V}$ при $D>0.2 \mathrm{Mм}$ и площади $S_{\mathrm{spec}}^{V}$ при $D>0.36$ мм практически не менялись при изменении $D$. Следовательно, в этой области $D$ плотность дефектов, средняя по накачиваемой области слоя, была практически неизменной. При $D \leq 0.2$ мм и $D \leq 0.36$ мм возникает некоторый разброс значений соответственно $S_{\mathrm{chr}}^{V}$ и $S_{\mathrm{spec}}^{V}$, вызванный изменением накачиваемого места образца (рис. 2). Следовательно, для таких малых $D$ средняя плотность дефектов несколько меняется в пространстве.

Сопоставим интенсивность излучения при разных $D$, какой бы она была, если бы $k$ при всех $D$ было одинаковым. Такая интенсивность, обозначим еe $I_{s}$, связана с измеренными хронограммами соотношением $I_{s}=a H_{s} / k S$, где $S=\pi D^{2} / 4, \quad(a-$ коэффициент, не зависящий от $D)$. Воспользуемся тем, что площадь под графиком хронограммы излучения $S_{\mathrm{chr}}=\int H_{s} d t$ можно представить как $S_{\mathrm{chr}}=b k l S W_{s}$, где коэффициент $b$, а также $l$ и $W_{s}$ - константы в условиях нашего эксперимента. Тогда для сравнения, не зависящего от $k$, интенсивность излучения можно представить в относительных единицах как $I_{s}=H_{s} / S_{\mathrm{chr}}$. В качестве примера на рис. 3 представлены графики $I_{s}=f(t)$ при $D=0.21$ и $1.1 \mathrm{Mм}$ и аналогично нормированной хронограммы накачки $I_{\mathrm{ex}}=H_{\mathrm{ex}} / S_{\mathrm{ex}}=f(t)$, где $S_{\mathrm{ex}}=\int H_{\mathrm{ex}} d t-$ площадь под графиком хронограммы накачки. Видно, что амплитуда зависимости $I_{s}(t)$ (обозначим амплитуду $\left.-I_{s-\max }\right)$ при $D=0.21$ мм больше, и релаксация излучения быстрее, чем при $D=1.1$ мм. Подробнее обнаруженное уменьшение максимальной интенсивности излучения при возрастании диаметра накачанной области иллюстрирует график $I_{s-\max }(D)$ (см. рис. 4). На рис. 4 представлены экспериментальные точки, для которых отклонение $S_{\mathrm{chr}}^{V}$ от среднего значения, показанного на рис. 2 сплошной линией, не превышало 50\%. На спаде излучение релаксирует с характерным временем $\tau_{r}$, зависимость которого от $D$ была выявлена в работе [1] и представлена на рис. 4 залитыми кружками. Обнаруживается антикорреляция между зависимостями $I_{s-\max }(D)$ и $\tau_{r}(D)$.

\section{3. Обсуждение результатов}

Из антикорреляции зависимостей $I_{s-\max }(D)$ и $\tau_{r}(D)$ (рис. 4), следует, что максимальную интенсивность из- 


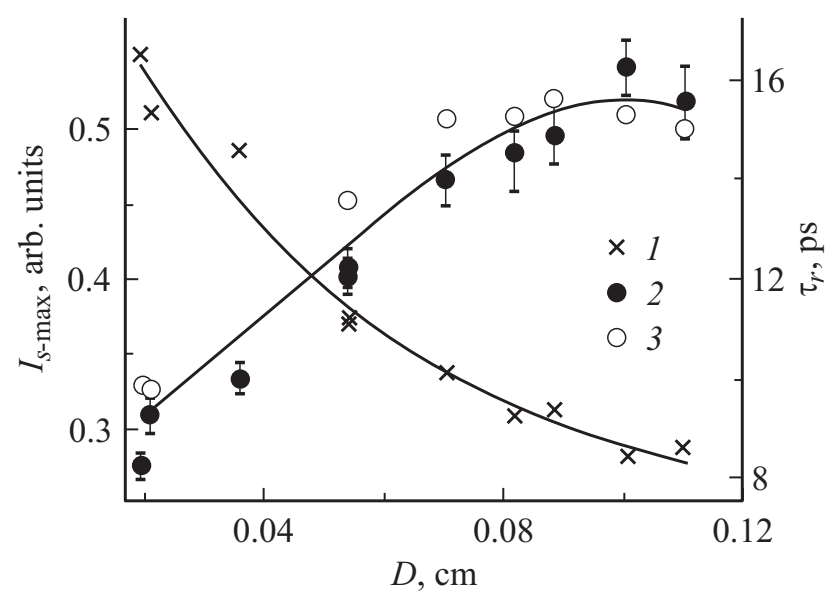

Рис. 4. Зависимость от диаметра $D$ максимальной интенсивности излучения $I_{s-\max }(1)$ и характерного времени релаксации излучения $\tau_{r}: 2$ - экспериментальные точки [1], 3 - расчет. Сплошные линии проведены для наглядности.

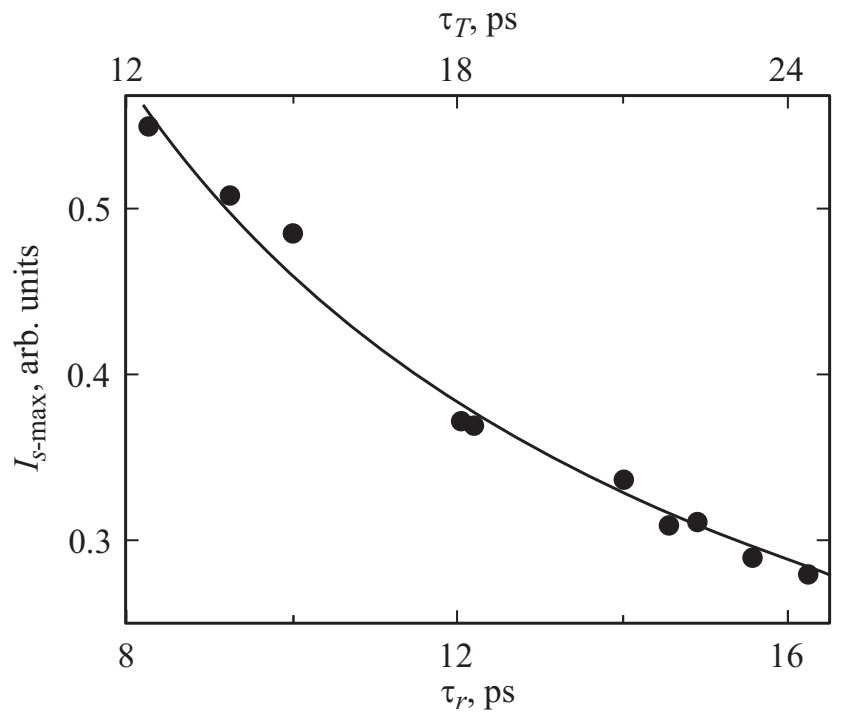

Рис. 5. Зависимость максимальной интенсивности излучения $I_{s-\max }$ от характерного времени релаксации излучения $\tau_{r}$ и температуры носителей заряда $\tau_{T}$. Сплошная линия - зависимость $I_{s-\max }=\beta / \tau_{r}(\beta=\mathrm{const})$.

лучения и характерное время его релаксации связывает соотношение

$$
I_{s-\max }=\beta / \tau_{r}
$$

где $\beta$ - коэффициент пропорциональности (рис. 5, нижняя ось абсцисс). Предположительно, можно объяснить антикорреляцию следующим образом.

Из-за взаимосвязи плотности и температуры носителей, возникающей при интенсивном излучении, время $\tau_{r}$ связано с характерным временем релаксации температуры (т. е. остывания) носителей $\tau_{T}$ соотношением [1]

$$
\tau_{r} \approx(2 / 3) \tau_{T}
$$

При определении $\tau_{T}$, исходя из измеренного $\tau_{r}$ и выражения (2), возникает обратно пропорциональная зависимость

$$
I_{s-\max }=3 \beta / 2 \tau_{T},
$$

представленная так же графиком на рис. 5, но с верхней осью абсцисс.

Как было обнаружено в работе [1] и иллюстрирует рис. 4, измеренное $\tau_{r}$ удовлетворительно согласуется с его оценкой, получаемой при подстановке в (2) аналитического выражения $\tau_{T}$, полученного в работе [6]. Это выражение учитывало замедление релаксации температуры носителей из-за разогрева носителей излучением:

$$
\tau_{T}=\left(A+B \tau_{p} T_{c}^{1 / 2} E_{g}\right) \tau_{h},
$$

где $A=6.3, B=0.4 ; \tau_{h} \approx 0.8$ пс - время релаксации энергии электронно-дырочной плазмы за счет эмиссии оптических фононов с учетом разогрева последних [7], $\tau_{T}, \tau_{p}, \tau_{h}$ измеряются в пс, средняя температура носителей $T_{c}-$ в $\mathrm{K}$, ширина запрещенной зоны $E_{g}-$ в эВ. Для лучшего согласия расчетной и экспериментальной зависимостей $\tau_{r}(D)$ (рис. 4) значения $A$ и $B$ незначительно изменены по сравнению с полученными в работе [6]: $A=6.2$ и $B=0.34$. Время $\tau_{p}-$ характерное время движения фотона излучения в активной среде, пока фотон не покинет эту среду или не будет поглощен:

$$
\tau_{p}^{-1} \approx c \chi^{-1}\left(\gamma+D^{-1}\right) .
$$

Здесь $\chi=3.6$ - показатель преломления $\mathrm{GaAs}$, $\gamma=\sigma n-$ коэффициент внутризонного поглощения света, $\sigma=1.5 \cdot 10^{-17} \mathrm{~cm}^{2}$ [8]. При оценке, выполнение которой в основном описано в работе [1], в выражения (4) и (5) подставляли параметры, соответствующие средней плотности электронов за время излучения $n_{\mathrm{av}}=\left(n_{m}+n_{\mathrm{th}}\right) / 2$, где $n_{\max }$ и $n_{\mathrm{th}}-$ максимальная и пороговая (при которой возникает излучение) плотности носителей заряда соответственно. Следует отметить, что плотность $n_{\max }$ при каждом $D$ определялась по ширине запрещенной зоны $E_{g}$, перенормированной из-за кулоновского взаимодействия носителей и соответственно зависевшей от их плотности. В настоящей работе значение $E_{g}$ определялось по длинноволновому краю нормированного спектра $W_{n}(\hbar \omega)=W(\hbar \omega) / S_{\text {spec }}$, где $S_{\mathrm{spec}}=\int W d(\hbar \omega)$. Нормировка на $S_{\mathrm{spec}}$ (аналогичная вышеописанной для хронограмм) приводит спектры к одинаковым $k$ и „энергии излучения“. В расчет принимались только те экспериментальные точки, для которых отклонение $S_{\text {spec }}^{V}$ от среднего значения не превышало $28 \%$.

При увеличении $D$ плотность носителей $n_{\max }$ уменьшалась, достигала минимума при $D_{b} \approx 0.7 \mathrm{MM}$, а затем возрастала (рис. 6). Такую зависимость $n_{\max }(D)$, предположительно, можно объяснить следующим. Интенсивность накачки была максимальной в центральной части накачиваемой области, и там она была приблизительно одинаковой при всех $D$. Пусть излучение движется с каждого участка наружной границы накачанной области 

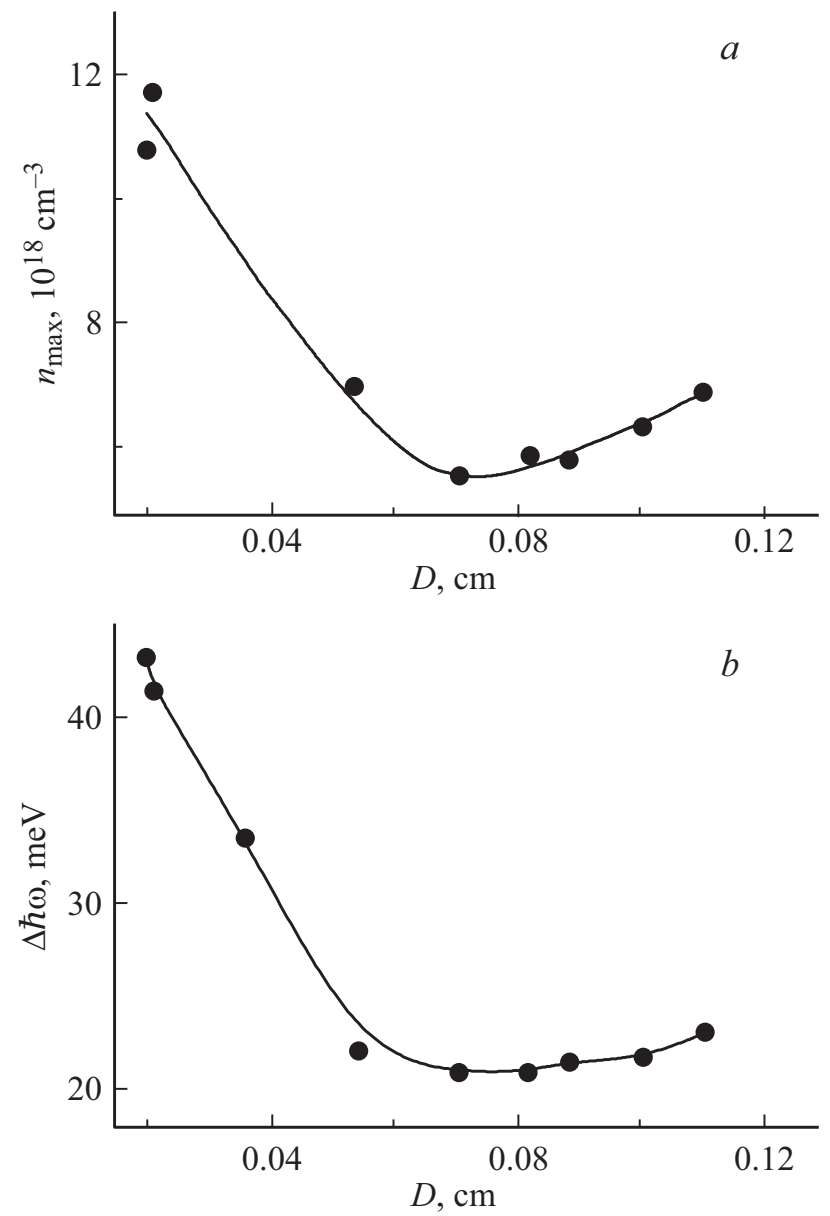

Рис. 6. Зависимость от диаметра $D$ максимальной плотности носителей $n_{\max }(a)$ и ширины спектра излучения $\Delta \hbar \omega(b)$. Сплошные линии проведены для наглядности.

по диаметру, бо́льшую часть пути в режиме насыщения усиления. Для одномерного случая насыщение рассмотрено в работе [3], для двумерного случая подобного рассмотрения мы не встречали. Все лучи, диаметрально пересекающие накачанную область, фокусируются в ее центре. Благодаря этому в центральной части накачанной области излучение имеет максимальную интенсивность. При увеличении $D$ в накачанной области будет возрастать разогрев носителей излучением, замедляющий их остывание [7]. Влияние этого замедления на скорость рекомбинации, среднюю по насыщенной области, оказывается сильнее, чем насыщающегося усиления излучения. Об этом свидетельствует уменьшение $I_{s-\max }$ при увеличении $D$. Но в центральной области скорость рекомбинации будет расти пока $D<D_{b}$. Этот рост вызван тем, что от той добавки к площади активной области, которая создается при увеличении $D$, возникает дополнительный вклад в интенсивность излучения в его фокусе. И пока $D<D_{b}$ этот вклад преобладает над вышеуказанным ослаблением излучения из-за возрастания разогрева носителей. Происходящее таким образом при увеличении $D$ до $D_{b}$ возрастание интенсивности излуче- ния, а значит, и скорости рекомбинации, в центре объясняет уменьшение $n_{\max }$. При $D>D_{b}$ вышеуказанное уменьшение интенсивности излучения из-за разогрева носителей становится доминирующим уже и в центре области. Из-за этого скорость стимулированной рекомбинации уменьшалась, а $n_{\max }$ соответственно возрастало, при увеличении $D$.

Обнаружилось, что ширина спектра излучения (FWHM) $\Delta \hbar \omega$ меняется с $D$ сходно с $n_{\max }(D)$ (рис. 6), демонстрируя связь ширины спектра усиления света и максимальной плотности носителей $n_{\max }$. Значительно более слабое возрастание $\Delta \hbar \omega$ с диаметром в области $D>D_{b}$ вызвано, по-видимому, тем, что вместе с $n_{\max }$ должна возрастать и максимальная температура носителей $T_{\max }$ в соответствии $\mathrm{c}$ их упоминавшейся взаимосвязью.

Учтем, что охлаждение носителей - это их внутризонные переходы (транспорт) на нижележащие энергетические уровни, и чем меньше $\tau_{T}$, тем интенсивнее (быстрее) энергетический транспорт носителей. Такой транспорт происходит и во время накачки носителей и после нее, пока релаксируют неравновесные носители. Когда интенсивность излучения настолько высока, что оно влияет на свое усиление (это так называемая стадия насыщения усиления), то увеличение $D$ при постоянной плотности энергии накачки приводит, как теперь можно предположить, к следующему. При каком-то $D$ достигается предельная скорость (средняя по накачанной области) энергетического транспорта носителей в область экстремумов зон проводимости и валентной (откуда они рекомбинируют) и стимулированной рекомбинации носителей. Соответственно достигается предел усиления излучения. Такой вывод позволяет сделать немонотонная зависимость $n_{\max }(D)$. Согласно остальным экспериментальным результатам этой работы и работы [1], при дальнейшем увеличении $D$ перечисленные скорости и усиление излучения уменьшаются. Это свидетельствует об автоматически поддерживаемом балансе между скоростью вынужденной излучательной рекомбинации носителей и энергетическим транспортом носителей. Последний замедляется из-за разогрева носителей излучением, увеличивающего время их охлаждения $\tau_{T}$. Баланс поддерживается автоматически излучением, путем регулирования обеднения инверсной заселенности электронами энергетических уровней зоны проводимости. Обеднение наблюдалось в работе [5].

\section{4. Заключение}

В итоге обнаружено следующее. Максимальная интенсивность собственного стимулированного пикосекундного излучения GaAs уменьшается при увеличении диаметра луча накачки $D$ (плотность энергии накачки фиксирована), что противоположно закону Бугера в линейной оптике. Эта зависимость антикоррелирует с зависимостью от диаметра $D$ характерного времени ре- 
лаксации излучения. Последнее пропорционально характерному времени остывания носителей заряда, которое поэтому тоже антикоррелирует с максимальной интенсивностью излучения. При увеличении $D$ максимальная плотность носителей $n_{\max }$ (в центральной части накачиваемой области) и ширина спектра излучения менялись с $D$ сходно немонотонно, что вызвано связью ширины спектра усиления света с максимальной плотностью носителей $n_{\max }$.

Обнаруженные явления возникают в так называемом режиме насыщения усиления, т.е. когда излучение влияет на его собственное усиление. Это подтверждается, например, обеднением инверсной заселенности электронами энергетических уровней зоны проводимости, создаваемым излучением, иначе говоря, создание провала в области усиления света в спектре поглощения, [5]. Обеднение служит для поддерживания баланса между скоростью вынужденной излучательной рекомбинации носителей и энергетическим транспортом носителей в область экстремумов зон проводимости и валентной, откуда они рекомбинируют. При увеличении $D$ возрастает разогрев носителей излучением, замедляющий их остывание и, соответственно, замедляющий их энергетический транспорт. При поддержании баланса между рекомбинацией и транспортом носителей замедление транспорта приводит к уменьшению интенсивности излучения и замедлению его релаксации. Таким образом, в работе показана роль энергетического транспорта носителей для генерации излучения в режиме насыщения его усиления. Переход от ненасыщенного режима к режиму насыщения усиления проявляется, в частности, в немонотонном изменении с $D$ плотности носителей $n_{\max }$.

Отметим, что выявлена еще одна важная при интенсивном пикосекундном излучении роль энергетического транспорта носителей, в данном случае для стимулированной рекомбинации. Ранее было обнаружено замедление из-за транспорта залечивания отклонений от фермиевского распределения носителей заряда [9] и создание LO-фононных осцилляций заселенности транспортом электронов, происходящим путем излучения LO-фононов $[5,10]$.

\section{Финансирование работы}

Работа выполнена в рамках государственного задания.

\section{Конфликт интересов}

Авторы заявляют об отсутствии конфликта интересов.

\section{Список литературы}

[1] Н.Н. Агеева, И.Л. Броневой, Д.Н. Забегаев, А.Н. Кривоносов. ФТП, (2019) (в печати).

[2] E.O. Goebel, O. Hildebrand, K. Lohnert. IEEE J. Quant. Electron., 13, 848 (1977).

[3] L.W. Casperson. J. Appl. Phys., 48, 256, (1977).
[4] Г.С. Ландсберг. Оптика (М., Наука, 1976).

[5] Н.Н. Агеева, И.Л. Броневой, А.Н. Кривоносов, С.Е. Кумеков, С.В. Стеганцов. ФТП, 36, 144 (2002).

[6] Ю.Д. Калафати, В.А. Кокин. ЖЭТФ, 99, 1793 (1991).

[7] С.Е. Кумеков, В.И. Перель. ЖЭТФ, 94, 346 (1988).

[8] J.S. Blakemore. J. Appl. Phys., 53, R123 (1982).

[9] Н.Н. Агеева, И.Л. Броневой, Д.Н. Забегаев, А.Н. Кривоносов. Радиотехника и электроника, 63, 1130 (2018).

[10] I.L. Bronevoi, A.N. Krivonosov, V.I. Perel'. Sol. St. Commun., 94 (9), 805 (1995).

Редактор А.Н. Смирнов

\section{Anticorrelation of the intensity of stimulated picosecond GaAs emission and the characteristic time of charge carriers cooling}

\author{
N.N. Ageeva, I.L. Bronevoi, D.N. Zabegaev, \\ A.N. Krivonosov \\ Kotel'nikov Institute of Radioengeneering and \\ Electronics, Russian Academy of Sciences, \\ 125009 Moscow, Russia
}

\begin{abstract}
During the powerful picosecond optical pumping, intense stimulated picosecond emission arises in a thin GaAs layer. It was found that, firstly, the maximum emission intensity decreases with increasing diameter of the pump beam (the pump energy density is fixed). Secondly, this dependence is anticorrelated with the dependence on the diameter of the characteristic relaxation time of the emission. And this time, in turn, is associated with the characteristic cooling time of charge carriers, which is slowed down due to heating of the carriers by emission. As a result, the autocorrelation indicated in the title is revealed.
\end{abstract}

